International Journal of Wireless \& Mobile Networks (IJWMN) Vol. 3, No. 4, August 2011

\title{
VERIFICATION OF ENERGY EFFICIENT Optimized Link State Routing Protocol USING PETRI NET
}

\author{
Radhika D. Joshi ${ }^{1}$ and Priti P.Rege ${ }^{2}$ \\ ${ }^{1}$ Department of Electronics and Telecommunication Engineering, College of \\ Engineering, Pune, India \\ rdj.extc@coep.ac.in \\ ${ }^{2}$ Department of Electronics and Telecommunication Engineering, College of \\ Engineering, Pune, India \\ ppr.extc@coep.ac. in
}

\begin{abstract}
Self-organizing ad hoc networks need development of efficient routing protocols in terms of reliable routing and energy conservation. For dense ad hoc networks, Optimized Link State Routing (OLSR) protocol is suitable due to its multipoint relaying $(M P R)$ feature. We have tried to make the OLSR energy efficient by making effective neighbor selection based on residual battery energy of a node and traffic conditions that influence the drain rate of the node in the network. We have considered the multipath and source routing concept for route selection and a route recovery technique to tackle mobility issue efficiently. Modifications make the protocol energy efficient and at the same time achieve balancing of network load. Simulation results of OLSR and the modified protocol, show improvement in 'Number of nodes alive' against variation in pause time, speed, node density and simulation time. The work describes modified protocol verification using Petri net for future instances.
\end{abstract}

\section{KEYWORDS}

MANET, OLSR protocol, multipath routing, Petri Net

\section{INTRODUCTION}

Wireless ad hoc networks are autonomous, self-configuring and adaptive. Thus, such networks are excellent candidates for military tactical networks, where their ability to be operational rapidly and without any centralized entity is essential [1]. The development of multimedia service brings new opportunities and challenges to the wireless network technologies.

In an ad hoc network, each node creates a network link in a self-organizing manner, forwarding data packets for other nodes in the network [2]. Mobile ad hoc networks (MANETs) are instantly deployable without any wired base station or fixed infrastructure [3]. Due to these features, MANETs suffers from limitations like lower capacity, limited security, higher loss rates, more delays and jitter as compared to fixed networks. A critical issue for MANETs is that the activity of node is energy-constrained [4]. In MANET, operations of nodes rely on batteries or other exhaustible power supplies for their energy. Hence depletion of batteries will have greater effect on overall network. As a consequence, energy saving is an important system design criterion. Furthermore, nodes have to be power-aware: the set of functions offered by a node depends on its available power (CPU, memory, etc.) [5]. Significant energy savings can be obtained at the routing level by designing minimum energy routing protocols that take into consideration the energy costs of a route when choosing the appropriate route. The Multipath routing protocols consist of finding multiple routes between a source node and a destination node. These multiple paths can be used to compensate for the dynamic and unpredictable nature of ad hoc networks [6].

DOI : 10.5121/ijwmn.2011.3413 
International Journal of Wireless \& Mobile Networks (IJWMN) Vol. 3, No. 4, August 2011

Though the major motivation of studying ad hoc networks comes from military usage, they will also be useful in several forms of tactical communication such as disaster recoveries, explorations, emergency services, law enforcements, educational applications, entertainment, location aware services and in various forms of home and personal area networks, as well as sensor networks $[7,8]$.

We present remainder of the paper as follows. In Section 2 we discuss issues of routing protocols and energy efficient routing protocol techniques in MANET. Original OLSR protocol features that are proposed to be modified are also discussed. Section 3 describes our scheme for making OLSR energy efficient. To modify existing protocol, various changes that are incorporated are illustrated in sub sections of Section 3. Section 4 includes simulation environment scenario used in NS-2 simulator. Section 5 shows performance comparison of OLSR and modified protocol, based on simulation results. The modified protocol verification using Petri net is illustrated in Section 6. Section 7 concludes the work focusing on, improvement in network behavior by using modified protocol, and verification of modified protocol using Petri net.

\section{LITERATURE SURVEY}

Routing protocols have to suggest best possible path from source to destination for efficient data transfer. For any application, the mobility of nodes as well as limited battery resources must be considered as design issues for expecting best performance from the network under consideration. It is very difficult to have correct data delivery under mobility conditions and to save the node power at the same time. Routing protocols are classified as Proactive, Reactive and Hybrid based on the method of maintaining route information in the protocol. In proactive protocols all routes are maintained regardless of the state of use [9].

Various techniques for making routing protocol energy efficient are considered. Saoucene Mahfoudh et al. [10] have distinguished three families of energy efficient routing protocols. Few proposals especially focused on the design of routing protocols providing efficient power utilization are dealt in depth by C.K.Toh [11]. The techniques are, Minimum Total Transmission Power Routing (MTPR), Minimum Battery Cost Routing (MBCR), Min-Max Battery Cost Routing (MMBCR), and Conditional Max- Min Battery Capacity Routing (CMMBCR). In addition to above techniques, minimum drain rate mechanism also needs to be considered for power saving. The drain rate is the rate at which energy gets dissipated at a given node. Each node monitors its energy consumption and maintains its battery power drain rate value during the given past interval.

Multipath routing offers several benefits like load balancing, fault-tolerance, higher aggregate bandwidth, lower end to end delay, reduced bottlenecks and security [12]. Multipath routing protocols have the advantage of sharing load of any flow on several paths, leading to a lesser consumption on the nodes of the selected paths. Multi-path routing techniques are proposed to use the minimized energy consumed per bit in discovering the least-required energy routing paths while reducing the computational complexity [13].Multiple paths can be formed for both traffic sources and intermediate nodes with new routes being discovered only when needed, reducing route discovery latency and routing overheads. Multiple paths can also balance network load by forwarding data packets on multiple paths at the same time [14].

\subsection{Selection of OLSR protocol for modification}

Optimized Link State Routing (OLSR) protocol selection is done from the view point of implementing multipath technique efficiently due to proactive nature of protocol. In proactive type of protocol energy management is the key issue. 
International Journal of Wireless \& Mobile Networks (IJWMN) Vol. 3, No. 4, August 2011

The main concept used in OLSR is that of multipoint relays (MPRs). MPRs are the selected nodes from one hop neighborhood which forward broadcast messages during the flooding process. This technique substantially reduces the message overhead as compared to a classical flooding mechanism (where every node retransmits each message received). In this way, nodes learn their local vicinity and the status of the link with each neighbor. The MPR set for a given node consists of a subset of neighboring nodes that covers the whole two-hop neighborhood of this node. Nodes learn their two-hop neighbor set by exchanging periodic HELLO messages.

This information is disseminated throughout the whole network via periodic Topology Control (TC) messages. This allows mobile nodes to set up routes to any potential destination present in the network. The TC message for a given node contains the set of nodes that have selected the sending node as an MPR. Once a node receives TC messages from other nodes, it can create routing directions to every node in the network using some sort of shortest path algorithm. This way a mobile host can reduce battery consumption.

OLSR provides optimal routes in terms of number of hops. The protocol is particularly suitable for large and dense networks as the technique of MPRs works well in this context. As a proactive protocol, OLSR is also suitable for scenarios where the communicating pairs change over time. No additional control traffic is generated in this situation since routes are maintained for all known destinations at all times [15]. The optimization achieved using the MPRs works well for large and dense mobile networks. The routing table is updated when a change is detected in either: the link set, the neighbor set, the 2-hop neighbor set, the topology set, or the Multiple Interface Association Information Base. Compared to On-Demand routing, proactive routing broadcasts more control messages in the network. If designed properly, these control messages could be effectively utilized to update the route table with only small overhead using multipath approach. In case of multi path OLSR, the routing table stores at the most two routes to every destination in the network. If the major route collapses, the other alternate route can be used immediately without another route discovery, thus, providing better Quality of Service (QoS) than the single route OLSR [16].

\section{MODIFICATION SCHEME USED TO MAKE OLSR ENERGY EFFICIENT}

We propose a modified protocol, including multipath and energy aware technique in OLSR. The modified protocol can be regarded as a hybrid multipath routing protocol. By using combined approach (multipath and energy aware technique), we expect a more fair distribution of the load along with even utilization of energy resources in the network so as to increase network lifetime as well as individual node lifetime in various dynamic conditions.

In order to include modifications in new protocol we have used reserved field available in the HELLO and TC packet format in original OLSR protocol, keeping all the remaining packets formats same. Reserved bits are modified by lifetime information in our modified protocol. Multipath source routing approach is used in association with the Min-Max Lifetime (MML) as an improvement over the conventional hop by hop routing in original OLSR protocol.

For the protocol modification, following changes are carried out at various stages:

\subsection{Calculation of willingness of MPR nodes}

An energy-aware selection of willingness can introduce an improvement in MPR selection, allowing the nodes to declare a willingness value of WILL_HIGH (meaning a high willingness to act as a MPR for its neighbors) or WILL_LOW (to signal a low willingness to forward neighbor's data). This way, a node can change its probability to be selected by its neighbors as a MPR, according to its own energy status [17]. In the default implementation of OLSR protocol, every node declares to its neighbors the same willingness (a value named WILL_DEFAULT). 
International Journal of Wireless \& Mobile Networks (IJWMN) Vol. 3, No. 4, August 2011

Thus in OLSR, each node has the same probability to be selected as a MPR by its neighbors, and the selection is performed only on the basis of the position of nodes.

In our protocol, the MPR selection is done when node energy is highest (W_High). If such node is not available, then node with medium energy (W_ Default) is selected. Node with less energy (W_Low) is never selected, even when it is the nearest node. It constructs an MPR-set that enables a node to reach any node in the symmetrical strict 2-hop neighborhood through relaying by one MPR node with willingness different from WILL_NEVER or WILL_LOW.

\subsection{Topology Sensing}

Due to correct MPR selection the flooding of broadcast packets in the network is avoided by reducing duplicate retransmissions in the same region. Multiple possible paths between source and destination pair are maintained instead of a single path. In order to take advantage of multiple paths we have increased TC packet frequency. This explores all possible paths to a particular destination. For our simulation, we have taken TOP_HOLD_TIME as 6 sec. TOP_HOLD_TIME [18] is given by Equation (1). For keeping track of movement of nodes we have reduced the TOP_HOLD_TIME, so that latest updates can be made available with less delay.

TOP_HOLD_TIME $=3 *$ TC_INTERVAL $\quad$--- Equation $(1)$

\subsection{Routes Computation}

The computation of routes uses the Multipath Algorithm to populate the multipath based on the information obtained from the topology sensing. In our modified protocol, routing table is calculated using Lifetime metric from the information maintained in neighbor set (accumulated from HELLO) \& topology set (accumulated from TC). Life time is predicted as ratio of residual battery energy to drain rate which is function of network traffic. The considerations for drain rate are according to current traffic conditions along with residual battery power. It provides a more optimized solution by considering the link traffic in an active network. The method used by each node to calculate the drain rate is similar to running average. Cost function is defined as the ratio of current remaining energy level to drain rate. The cost function is inversely proportional to the network resources used, if the data transmission is to be carried out by that node to its neighbors. This function is then added to the TC as well as to the HELLO packet. The destination node now selects the path in which the least cost function is highest among a set of routes in routing table, leading to destination. The route request packet consists of an IP header.

\subsection{Source Routing in modified protocol}

In OLSR, due to next hop routing, node can forward data based on its own routing table. It may not get the correct next node from the nodes available to reach the destination. To avoid the problem for the next hop routing in standard OLSR protocol, we use the source path in our modified algorithm. In the routing table of our protocol, information related to hops from destination to source is stored. Source routing will help the source node to keep good control of the packets which will be forwarded in the multipath.

\subsection{Route Recovery}

In the classical OLSR, the hop-by-hop routing is used, which means when a packet reaches an intermediate node, the protocol will check the routing table of the local node and then forward the packet to the next hop. However, in the mean time, the pure source routing might cause two problems: 
International Journal of Wireless \& Mobile Networks (IJWMN) Vol. 3, No. 4, August 2011

- Firstly, the information in the source node might not be the latest because it needs time to flood the topology control messages to the whole network, i.e. while computing the routes; the source node might use the links that do not exist anymore.

- Secondly, even when the information in the source node is updated, the topology might change during the forwarding of the packet.

Both situations will cause the failure of the packet forwarding.

To overcome this problem, we carry out route recovery as explained in step 6 of Implementation for modified Algorithm. Functional representation of the modified protocol is given in Figure 1.

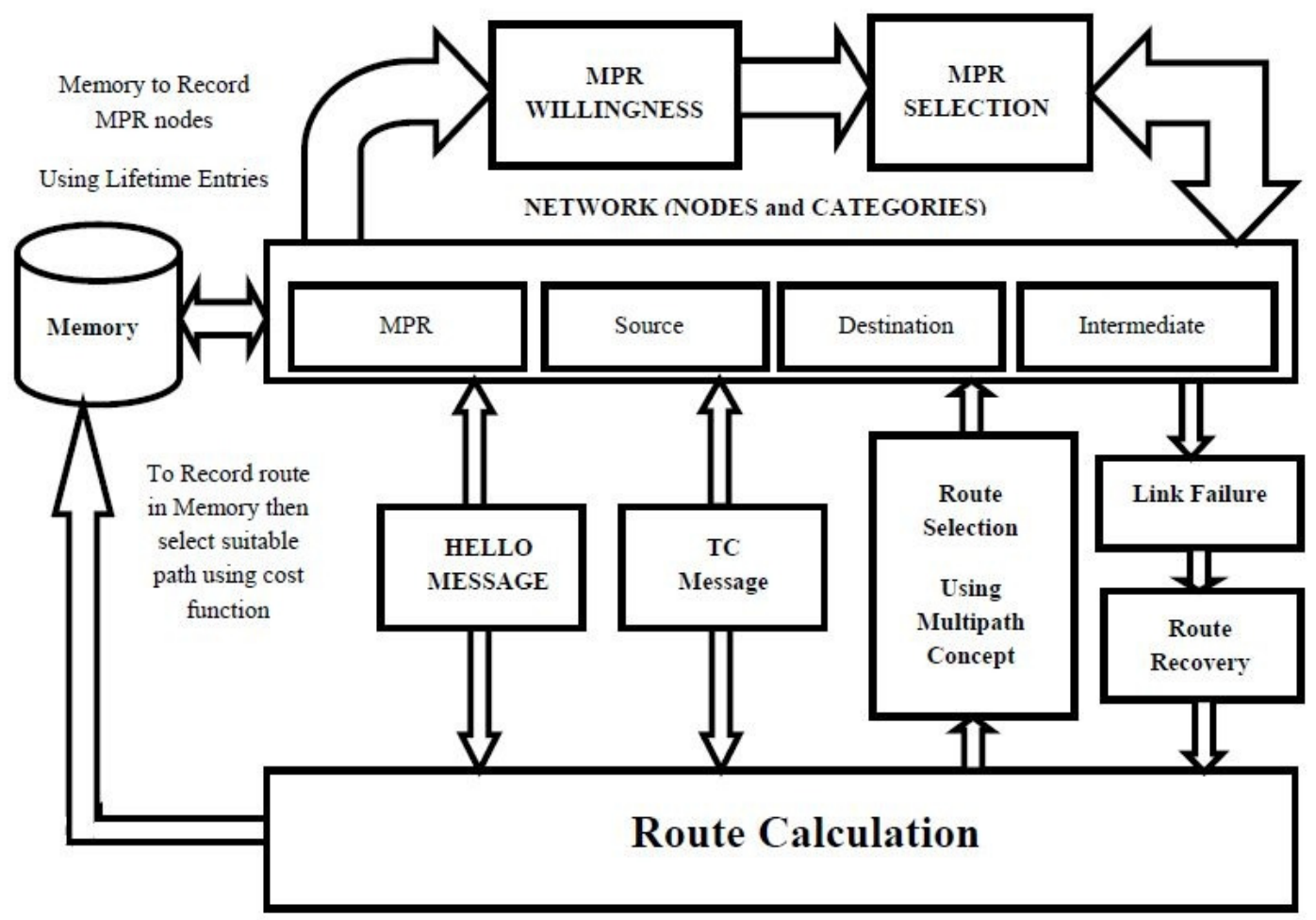

Figure 1. Functional representation of the modified protocol

\subsection{Implementation steps for making OLSR energy efficient}

1. Remove all previous entries from MPR table.

2. Selection of MPR based on lifetime calculation showing impact of willingness.

3. Based on its neighbor table; insert the new entry to its routing table along with lifetime information.

4. Insert the new entry to routing table along with lifetime values of nodes on path and record the complete path information in the routing table.

5. Perform Route calculation based on following steps:

a. Remove all previous entries for route.

b. Add new routing entries with symmetric neighbours as intermediate single-hop neighbours.

c. Record the new route entry by incrementing hop count by one each time till the destination node is reached.

d. Records several route entries for same destination with different cost values i.e. multiple paths are stored in memory for single destination called multipath routing. 
International Journal of Wireless \& Mobile Networks (IJWMN) Vol. 3, No. 4, August 2011

e. Out of these multiple paths, select the one with minimum cost value for delivering message to the destination.

As our network is mobile, each node changes its position periodically so that there may be possibility of link breakages. Hence, the concept of route recovery is introduced to get another route to destination.

6. Route Recovery: For intermediate node, check if the next hop in the source route of the packet is one of its neighbors, before trying to forward the packet,

- If yes, then forward the packet as it is.

- If no, then recompute the route to destination and then forward the packet through the new route (This represents the case when the "next hop" has moved out of the transmission range of the node).

\subsection{Performance criteria}

We use the metric 'Number of nodes alive' in our simulation trials. This metric indicates the overall lifetime of the network [19]. In wireless Ad Hoc networks, especially in those with densely distributed nodes, the death of the first node seldom leads to the total failure of the network. With number of dead nodes increasing, the network is likely to partition. More importantly, it gives an idea of the area coverage of the network over time. Network lifetime is one of important metrics to evaluate the energy efficiency of the routing protocols with respect to network partition [20].

\section{Simulation}

Simulation is carried out for OLSR and modified protocol. Ns-2 is used to compare ad hoc routing protocols over Random waypoint mobility models. The underlying MAC layer protocol is defined by IEEE 802.11 standard [21].

We have measured 'Number of nodes alive' for both the protocols. We observed the effect of variation of pause time, speed and number of nodes on both the protocols. As we are considering node mobility issue, both, speed and pause time play major role. Depending upon their values, the nodes move making the scenario dynamic, leading to few path breaks. Thus the route recovery feature gets tested. Node density shows the network state, sparse or dense, and whether it affects protocol behavior.

Simulations are carried out for three input parameter variations. They are listed at the top of three columns in Table I. The variable parameter entry in a column is varied only for that particular parameter. Other parameter values are same for the three cases.

We have considered field size as $670 * 670$ sq. meters by referring most of simulation trials to earlier work. In order to have sufficient observation interval, simulation time is selected as 1000s. From our previous trial experience we have selected total nodes present in the network as 35 [22]. Number of connections among the nodes is usually half the node count and accordingly the value is selected. A medium data packet rate is selected for the connections. Initial node energy and communication range are interrelated. If node energy is more, then communication range can be large and vice versa. We have selected moderate values of these parameters.

\subsection{Considerations for mobility model used for simulation}

The performance of ad hoc routing protocols greatly depends on the mobility model used. Random Waypoint is considered to be an entirely random scheme and intuitively can be the most challenging environment for ad hoc routing protocols [23]. Most of the multipath protocols \& the energy aware protocols are analyzed in static environment. We analyze our protocol using random way point mobility model which provides worst case test conditions with various dynamic situations. 
International Journal of Wireless \& Mobile Networks (IJWMN) Vol. 3, No. 4, August 2011

\section{SIMULATION RESULTS FOR MODIFIED PROTOCOL AND OLSR}

We observed the effect of number of nodes, speed and pause time variation on both the protocols' simulated parameters. Number of nodes i.e. Node density for fixed area shows the network state (sparse or dense) and how it affects protocol behavior. Number of connections among the nodes should be half that of number of nodes, and should be changed as per nodes under consideration, for the trial. Network mobility effect can be studied by varying speed and pause time. For the trials, we have varied one parameter at a time, keeping other parameters constant. Simulations scenario is given in Table 1.

Table 1. Parameter selection used for the simulation in Ns-2

\begin{tabular}{|l|l|l|l|}
\hline Parameters & $\begin{array}{l}\text { Pause time } \\
\text { (seconds) }\end{array}$ & Speed (meter/sec.) & Number of nodes \\
\hline Number of mobile nodes & 35 & 35 & $10,20,30,40,50,60$ \\
\hline Field size (m) & $670 * 670$ & $670 * 670$ & $670 * 670$ \\
\hline Simulation duration (s) & 1000 & 1000 & 1000 \\
\hline Number of connections & 17 & 17 & $5,10,15,20,25,30$ \\
\hline Speed (m/s) & 5 & $1,5,10,15,20,25,30$ & 5 \\
\hline Pause time (s) & $\begin{array}{l}50,100,150,200, \\
250,300,350,400\end{array}$ & 100 & 100 \\
\hline Mac Layer & IEEE 802.11 & IEEE 802.11 & IEEE 802.11 \\
\hline $\begin{array}{l}\text { Communication range } \\
\text { (m) }\end{array}$ & 100 & 100 & 100 \\
\hline $\begin{array}{l}\text { Data packet rate for each } \\
\text { connection (packets/s) }\end{array}$ & 20 & 20 & 20 \\
\hline Initial node energy (J) & 10 & 10 & 10 \\
\hline Mobility Model & Random Waypoint & Random Waypoint & Random Waypoint \\
\hline
\end{tabular}

The comparative analysis of both the protocols for energy related parameter as Number of nodes alive is given in the following sections.

\subsection{Effect of pause time}

In simulation, pause time can vary from minimum zero sec to half of simulation time. For our simulation purpose we have varied the pause time from 50 to $400 \mathrm{sec}$. The impact of pause time on Number of nodes alive is tested.

The comparison of Number of nodes alive versus pause time variation for both the protocols is given in Table 2. It is observed that our protocol shows small improvement in Number of nodes alive as compared to OLSR for the pause time values ranging between $150 \mathrm{sec}$ to $350 \mathrm{sec}$.

Table 2. Effect of pause time variation on Number of nodes alive.

\begin{tabular}{|l|l|l|}
\hline Pause Time (sec) & $\begin{array}{l}\text { Number of nodes } \\
\text { alive - OLSR }\end{array}$ & $\begin{array}{l}\text { Number of nodes alive } \\
\text { - Modified protocol }\end{array}$ \\
\hline 50 & 0 & 0 \\
\hline 100 & 1 & 1 \\
\hline 150 & 4 & 7 \\
\hline 200 & 10 & 11 \\
\hline 250 & 11 & 13 \\
\hline 300 & 13 & 15 \\
\hline 350 & 14 & 16 \\
\hline 400 & 23 & 23 \\
\hline
\end{tabular}




\subsection{Effect of speed}

During simulation, we have considered speed variation from $1 \mathrm{~m} / \mathrm{s}$ to $30 \mathrm{~m} / \mathrm{s}$ in steps of $5 \mathrm{~m} / \mathrm{s}$, to demonstrate different node movement cases. The lowest speed represents person walking, speed as $1 \mathrm{~m} / \mathrm{s}$ i.e. $3.6 \mathrm{~km} / \mathrm{hr}$ and other case is that of a moving vehicle with speed $30 \mathrm{~m} / \mathrm{s}$ or 108 $\mathrm{km} / \mathrm{hr}$ for fast mobility applications. The considerations are based on Indian scenario.

The Number of nodes alive condition is better maintained for the modified protocol. It is observed that at medium speed i.e. at $15 \mathrm{~m} / \mathrm{s}$ modified protocol performs better as compared to OLSR as seen in Table 3 .

Table 3. Effect of speed variation on Number of nodes alive

\begin{tabular}{|l|l|l|}
\hline Speed (meter/sec) & $\begin{array}{l}\text { Number of nodes } \\
\text { alive - OLSR }\end{array}$ & $\begin{array}{l}\text { Number of nodes alive } \\
\text { - Modified protocol }\end{array}$ \\
\hline 1 & 32 & 32 \\
\hline 5 & 19 & 20 \\
\hline 10 & 20 & 21 \\
\hline 15 & 15 & 22 \\
\hline 20 & 17 & 18 \\
\hline 25 & 15 & 15 \\
\hline 30 & 8 & 13 \\
\hline
\end{tabular}

\subsection{Effect of number of nodes}

When topography under consideration is kept constant and we vary number of nodes from 10 to 60 in steps of 10 nodes, the effect of nodes variation on Number of nodes alive is observed. It is observed from Table 4, that, modified protocol shows improvement in Number of nodes alive as compared to OLSR when network contains more number of nodes i.e. 60 nodes. This confirms the effective use of our protocol for dense networks.

Table 4. Effect of number of nodes variation on Number of nodes alive

\begin{tabular}{|l|l|l|}
\hline Number of Nodes & $\begin{array}{l}\text { Number of nodes } \\
\text { alive - OLSR }\end{array}$ & $\begin{array}{l}\text { Number of nodes alive } \\
\text { - Modified protocol }\end{array}$ \\
\hline 10 & 6 & 6 \\
\hline 20 & 15 & 16 \\
\hline 30 & 17 & 17 \\
\hline 40 & 18 & 18 \\
\hline 50 & 19 & 20 \\
\hline 60 & 20 & 25 \\
\hline
\end{tabular}

The protocol testing is further carried out for Simulation time variation for two sample values of pause time, speed and number of nodes. These trials help to decide better choice of these three parameters for network performance.

\subsection{Simulation time variation for two cases of pause time}

Two sample values of Pause time (250 and $400 \mathrm{sec})$ are selected from the range 50 to $400 \mathrm{sec}$ for simulation time variation trials. We have carried out simulations for twice the selected pause time value. The comparison of protocols for both cases, for same simulation time duration is given in Figure 2. The results show modified protocol is superior as compared to OLSR for both pause time conditions, from $300 \mathrm{sec}$ simulation time. Number of nodes alive is more for $400 \mathrm{sec}$ as compared to $250 \mathrm{sec}$. 
International Journal of Wireless \& Mobile Networks (IJWMN) Vol. 3, No. 4, August 2011

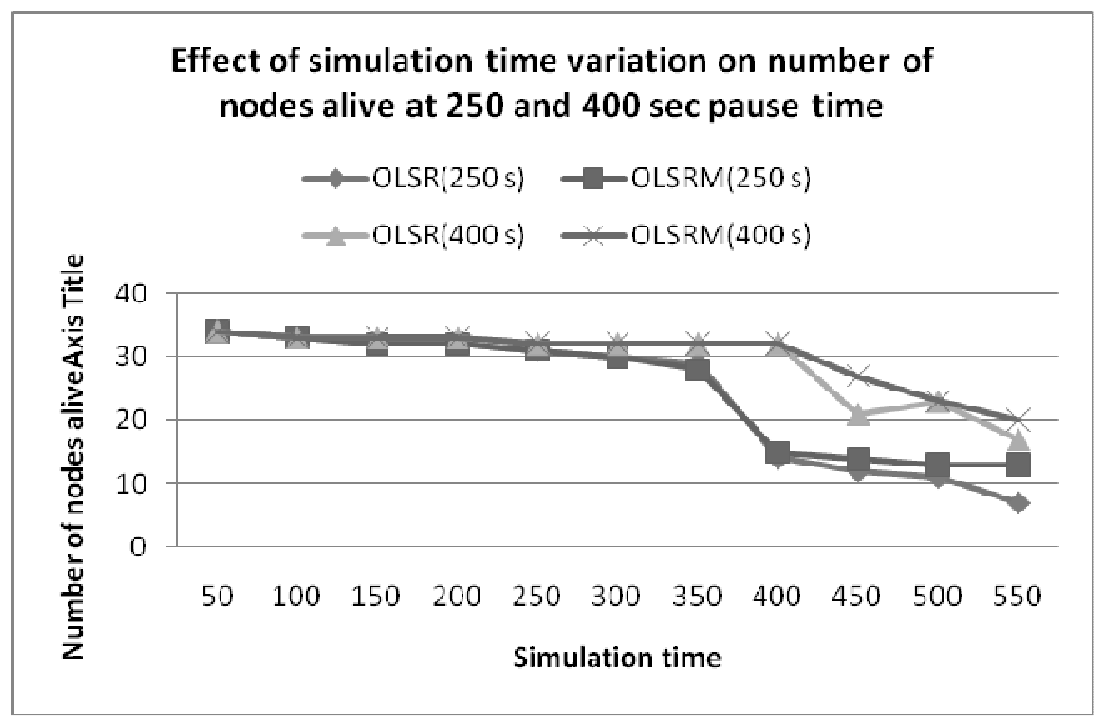

Figure 2. Effect of simulation time variation on number of nodes alive, for OLSR and modified protocol alive at $250 \mathrm{sec}$ and $400 \mathrm{sec}$ pause time

\subsection{Simulation time variation for two cases of speed}

Number of nodes alive against simulation time at $1 \mathrm{~m} / \mathrm{s}$ and $15 \mathrm{~m} / \mathrm{s}$ speed for OLSR and modified protocol is given in Figure 3. No significant difference is observed in number of node alive, for both protocols except for simulation time as 450 and $500 \mathrm{sec}$ for both speed conditions.

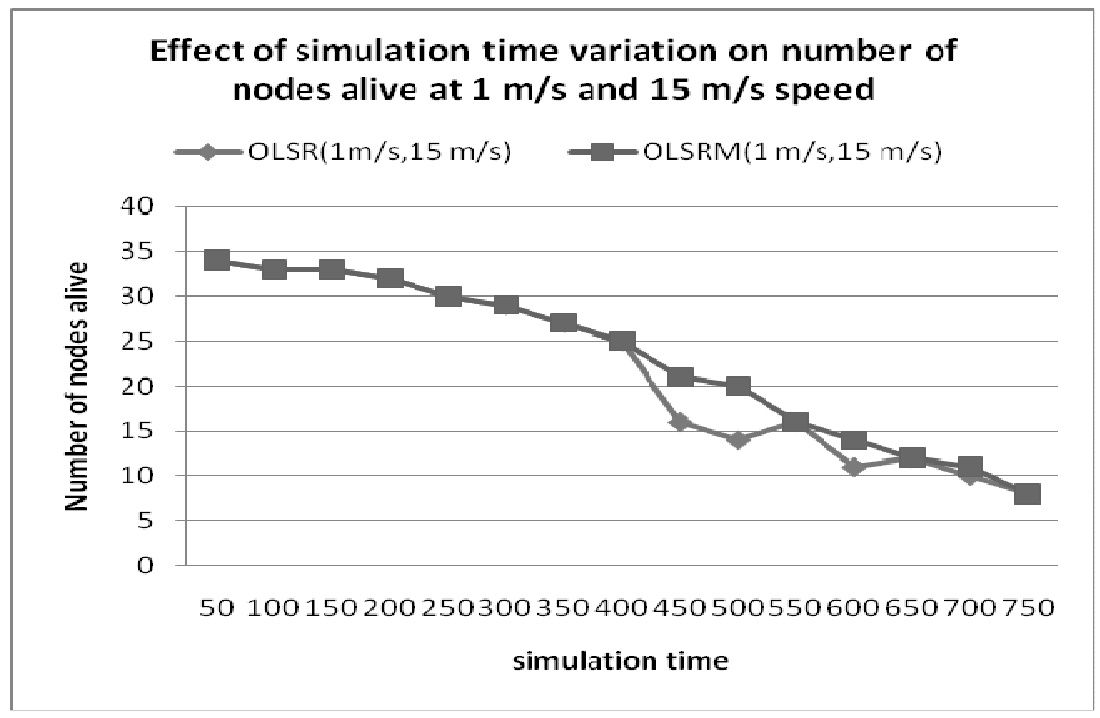

Figure 3. Effect of simulation time variation on number of nodes alive, for OLSR and modified protocol at $1 \mathrm{~m} / \mathrm{s}$ and $15 \mathrm{~m} / \mathrm{s}$ speed conditions

\subsection{Simulation time variation for two cases of number of nodes}

Number of nodes alive against simulation time with 40 and 60 nodes for OLSR and modified protocol is given in Figure 4. When number of nodes selected for given scenario is more, initially when simulation starts, the node count decreases earlier. Later, over total simulation time trials, Number of nodes alive for modified protocol is better. 
International Journal of Wireless \& Mobile Networks (IJWMN) Vol. 3, No. 4, August 2011

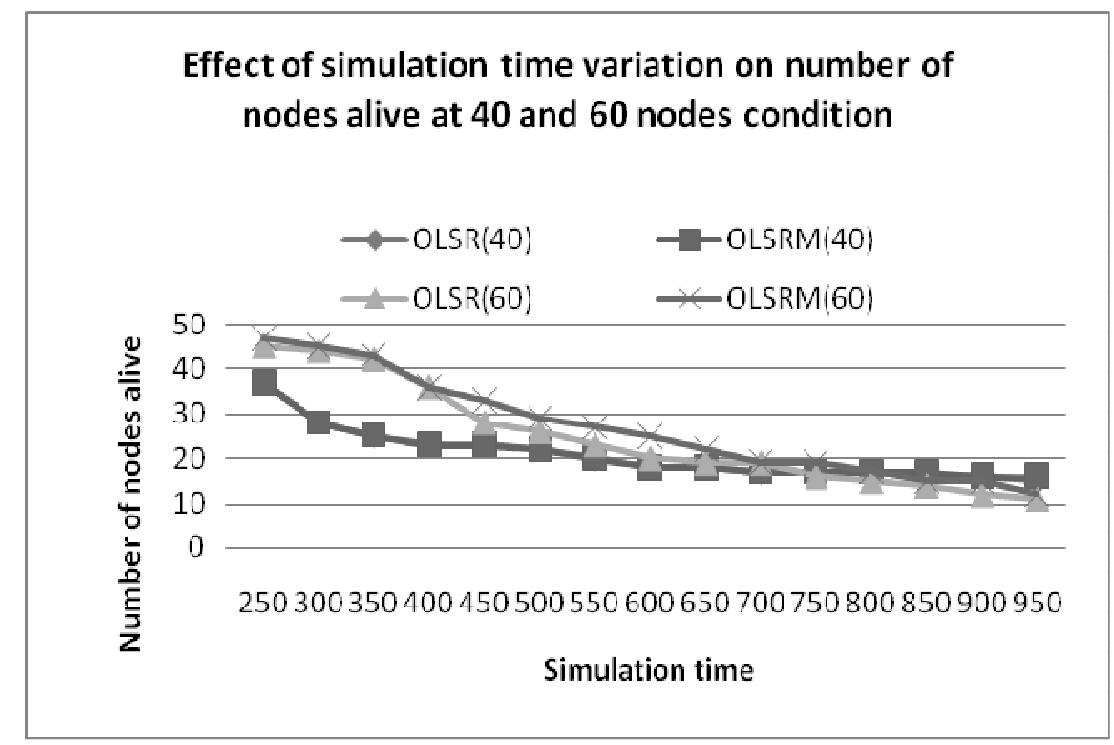

Figure 4. Effect of simulation time variation on number of nodes alive, for OLSR and modified protocol at 40 and 60 number of nodes conditions

\section{Petri Net}

The main benefit of modeling is that it provides insight about the properties of the system prior to implementation. This allows many issues about the system to be resolved in the design phase rather than in the implementation phase.

The use of formal description techniques results in models that are amenable to verification. An advantage of many formal description techniques is that, they are based on the construction of executable models that make it possible to observe and experiment with the behavior of the protocol prior to implementation using simulation. This typically leads to complete specifications since the model will not be fully operational until all parts of the protocol have been specified. Another advantage of formal modeling languages is that they support abstractions, making it possible to specify the operation of the protocol without being concerned with irrelevant implementation details. The complex behavior of communication protocol makes the design of correct protocols a challenging task due to number of independent concurrent protocol entities.

Petri nets are a fundamental, visual and formal modeling technique in concurrency and have been subject for suitable extensions in particular to model reconfigurations of the net structure and the exchange of data. Thus they are profitably applied to model workflows in mobile ad-hoc networks and flexible communication based systems [24]. Petri net (PN) have an appealing conceptual simplicity based on graphical representation of the mechanism of process interaction. Due to the dynamic nature, concurrency and different levels of abstraction associated with the Mobile Ad-Hoc Network (MANET) protocols, Colored Petri Nets (CPN) is a suitable modeling language for this purpose. This is a promising tool for describing and studying information processing systems that are characterized as being concurrent, asynchronous, distributed, parallel, nondeterministic and stochastic [25]. Petri net being state transition based model can be used widely for protocol studies for handling concurrent or communication systems. PN provides only a structural description of protocols; the dynamic aspects of protocol like control and data flow are described in terms of firing rules and token distribution [26]. High level Petri nets are powerful for reliable specification for translation of requirement definitions into verified specifications. High level Petri net goes 
International Journal of Wireless \& Mobile Networks (IJWMN) Vol. 3, No. 4, August 2011

through various phases such as requirement definition, validation, specification and implementation [27].

$\mathrm{CP}$-nets or CPNs is a formal modeling language that is well suited for modeling and analyzing large and complex systems for several reasons: hierarchical models can be constructed, complex information can be represented in the token values and inscriptions of the models, timing information can be included in the models, and mature and well-tested tools exist for creating, simulating, and analyzing CPN models [28]. CP-nets are often used to model and verify the logical correctness of network protocols. Colored Petri nets provide a framework for the design, specification, validation, and verification of systems. CP-nets have a wide range of application areas like communication protocols, operating systems, hardware designs, embedded systems, software system designs, and business process re-engineering. Design/CPN is a graphical computer tool supporting the practical use of CP-nets [29]. The tool supports the construction, simulation, functional and performance analysis of CPN models. CPN models can be structured into a number of related modules. The module concept of CP-nets is based on a hierarchical structuring mechanism (either top down or bottom up approach). CP-nets include a time concept which makes it possible to capture the time taken by different activities in the system. Timed CPN models and simulation can be used to analyze the performance of a system, by investigating Quality of Service (QoS) parameters like, delay, and throughput. It is possible to investigate the functional correctness of systems modeled by means of timed CP-nets. Abstract CPN models can be used in an early phase of system development to determine the boundaries of the project and specify requirements [30]. CP-nets have a sound, mathematically well founded execution semantics, are well-proven, and have proper tool support. The design and specification can be supported by modeling and simulation using Hierarchical Colored Petri Nets (CP-nets). The main purpose of these models has been to analyze the behavior of existing systems, it can be considered as an integrated part of the design phases (which is more time consuming than actual coding) of the development of distributed software systems. The use of $\mathrm{CP}$-nets in the design phase contributed to the development of a better product using fewer resources [31]. Hierarchical nets provide construction of complicated models [32]. In such nets an element may be represented by another net i. e. nested construction: net inside net. The number of hierarchy levels has no principal limitations like programming languages where procedures are used to maintain the complexity.

The CPN modeling language and supporting computer tools are powerful enough to specify and analyze a real-world communication protocol [33]. The act of constructing the CPN model, executing, and discussing it lead to the identification of several non-trivial design errors and issues that under normal circumstances would not have been discovered until at best in the implementation phase.

\subsection{Verification of modified energy efficient OLSR protocol using Hierarchical Colored Petri Nets (HCPN)}

Main-body As protocol modification is done at different parts, we have used HCPN technique. Figures 6 to 8 illustrate separate mechanisms that are used to build modified OLSR as given in Figure 5. For practical reasons it is not desirable to create a single large CPN that specifies a given firewall system in a flat structure. The concept of Hierarchical CPNs allows a designer to construct large CPNs by combining a number of smaller CPNs. They are beneficial for the modular composition of CPNs. HCPNs can be constructed top-down, bottom-up, or by a mixture of these two strategies. We are using top down approach for HCPN. HCPNs make it possible to relate a number of individual CPNs to each other in a formal way, and thus allow their formal analysis. In a top-down design one starts with a simple high level description of a system without consideration for internal details. A specification of detailed behavior of the CPN is developed through stepwise refinement. Stepwise refinement is achieved through the application of a construct called substitution transition, where a more complex CPN takes the place of a transition. The CPN must conform to the interface of the replaced transition and relate 
identically to its surrounding arcs. In a bottom-up design CPNs are combined into a larger net through fusion places. A fusion place is a set of places that are considered to be identical. Even if they are drawn as individual places they represent a single conceptual place. For each token that is added (removed) at one of the places, an identical token is added (removed) at all other places. Point ' $A$ ' in Figure 6 and in Figure 7 is a fusion place, as it appears in both places identically. A non-hierarchical CPN is called a page. A page that contains a substitution transition is called a superpage as shown in Figure 6. A page that contains the detailed description of the activity modeled by the corresponding substitution transition is called subpage given in Figure 5. A substitution transition is also called a supernode. Note that the places connected to a substitution transition by a single arc (called socket nodes) and their counterparts on the subpage (called port nodes) are fusion places. The interface between a superpage and a subpage is defined through port assignments where socket nodes are related to port nodes.

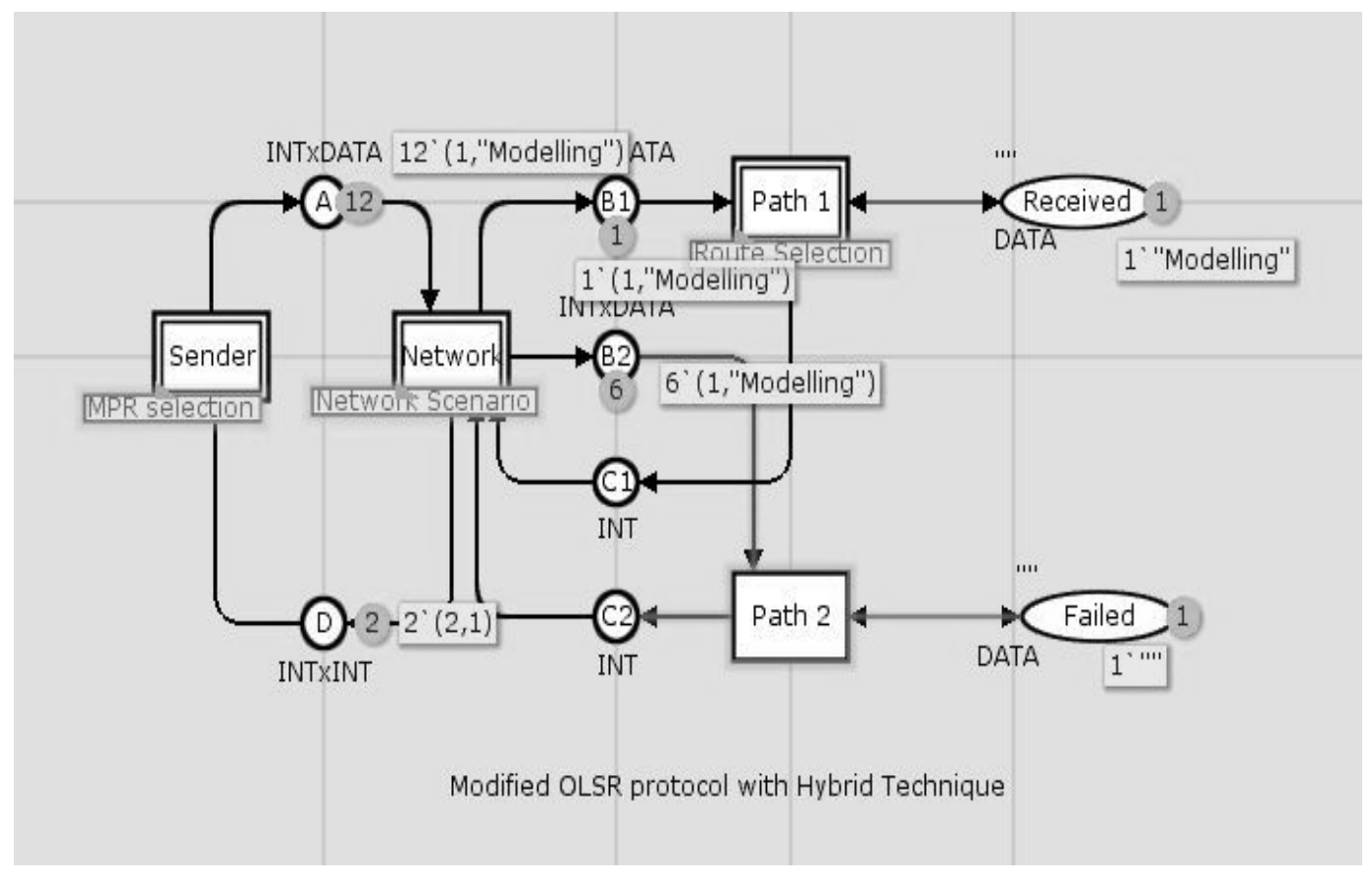

Figure 5. Modified protocol with Hybrid Technique represented in CPN

\subsection{Working}

Figure 5 shows whole functioning of Modified OLSR protocol, which is further divided into three parts.

\subsubsection{MPR Selection criteria (Figure 6) \\ 6.2.2 Network Scenario with multipath selection (Figure 7) \\ 6.2.3 Energy Efficient Route Selection (Figure 8)}

HCPN represented by Figure 5, describes an example of Modified OLSR that combines MPR selection and Route Selection mechanisms. We structure the description top-down, starting with the superpage. Sender sends a packet to the Network by creating a copy of the packet on place A. It should be noted that neither the packet is removed from Send nor the counter at Next Send increased. The reason for retaining the packet is that, the packet may be lost and hence should be retransmitted. Our protocol continues to repeat the same packet until it gets an acknowledgement telling that the packet has been successfully received. Transition shows a packet transmits from the Sender site of the Network to the Receiver site by comparing cost 
International Journal of Wireless \& Mobile Networks (IJWMN) Vol. 3, No. 4, August 2011

value for different paths and moving the corresponding token from A to B. It should be noted that the Route Selection page is used by two substitution transitions, Received and Failed. This means that we will have two instances of the subnet - during a simulation. The two instances may have different markings and different enabling depending on route selection.

\subsubsection{MPR Selection}

The MPR selection part is used to select node as MPR depending on WILLINGNESS. The only difference is that Receive Acknowledgment now needs an acknowledgment from Receiver in order to become enabled.

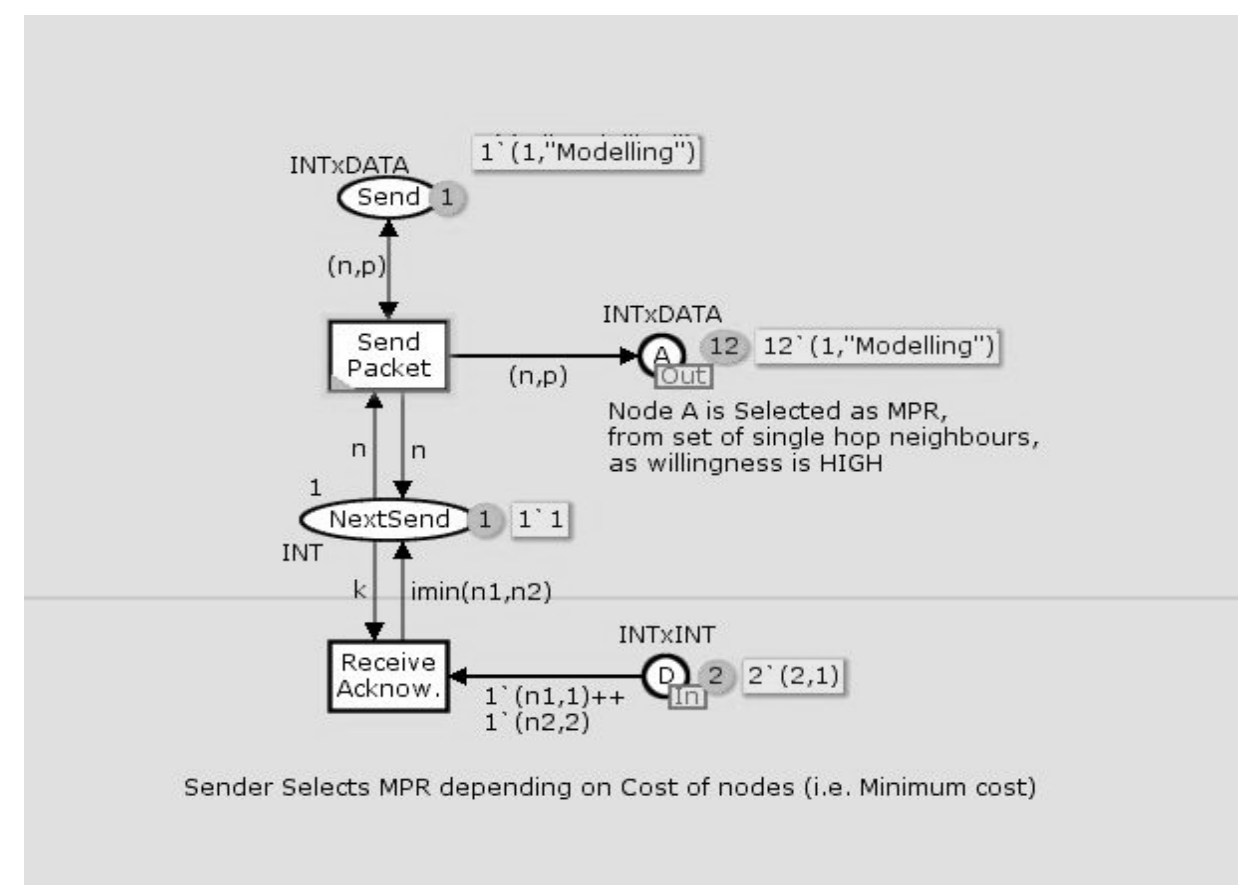

Figure 6. MPR Selection Criteria based on node cost

\subsubsection{Network Scenario with multipath option}

Transmit Packet produces packets at two different output places B1 and B2 (i.e. nodes). The packets at B1 and B2 are for the Receiver. It should be noted that we use two different variables r1 and $\mathrm{r} 2$ to determine whether the packets for B1 and B2 are lost or not. This means that we model a broadcast in which one of the paths may get a packet while the other does not. Transmit Acknowledgment is split in two parts in case of path failure. The Transmit acknowledgment can be from $\mathrm{C} 1$ on the path (B1) or from $\mathrm{C} 2$ on the path (B2). B1 and B2 modify the acknowledgment, by adding information telling the Sender from where the acknowledgment came. The Sender selects energy efficient path among B1 and B2. The Boolean expression Ok $(\mathrm{s}, \mathrm{r})$ determines whether the packet is successfully transmitted or lost. Each acknowledgment is a pair $(\mathrm{C} 1, \mathrm{~B} 1)$ or $(\mathrm{C} 2, \mathrm{~B} 2)$ where the first element is contents, while the second element indicates Path as shown in Figure 7. 
International Journal of Wireless \& Mobile Networks (IJWMN) Vol. 3, No. 4, August 2011

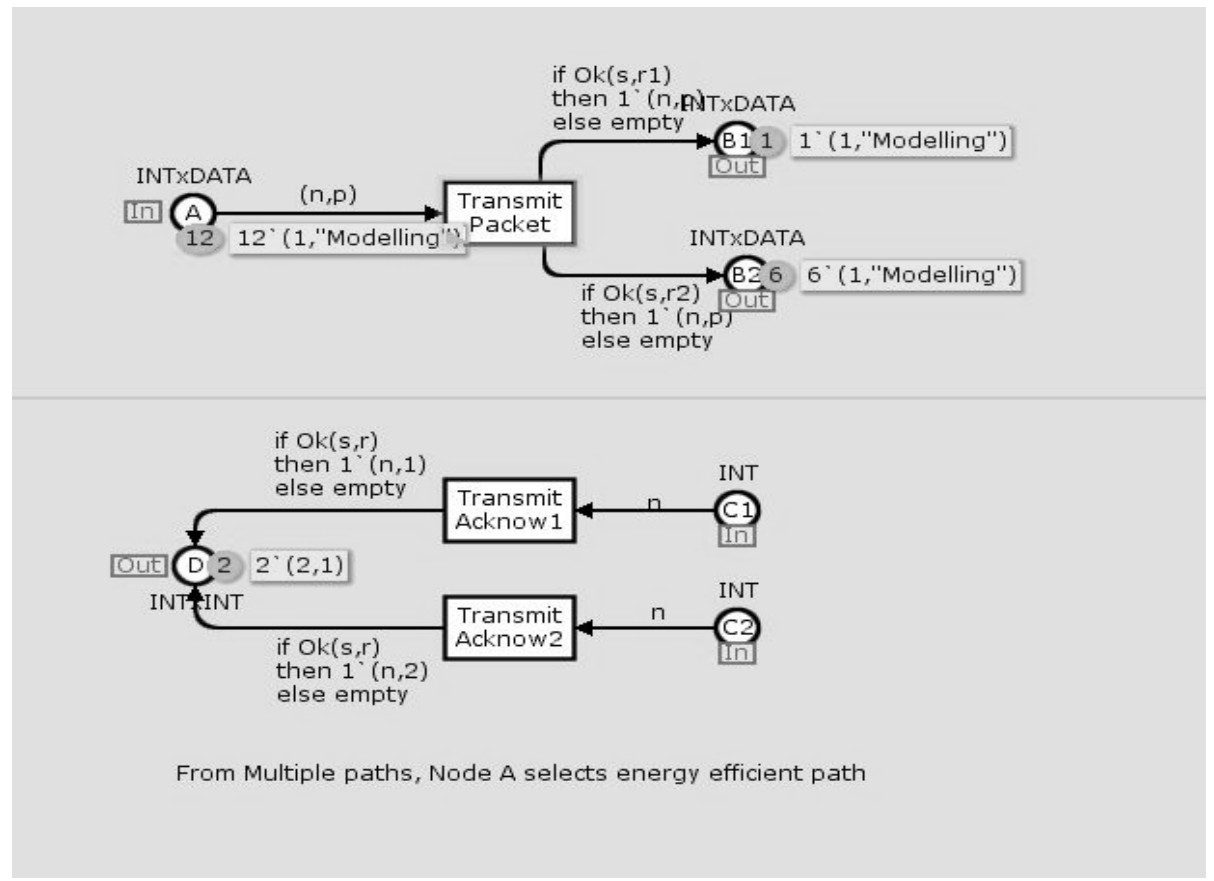

Figure 7. Network Scenario with Multipath option

\subsubsection{Energy Efficient Route Selection}

Path having minimum cost is selected for transmission. The desirable path B1 is denoted by B; similarly contents $\mathrm{C} 1$ are denoted as $\mathrm{C}$. This is route selection criteria shown in Figure 8.

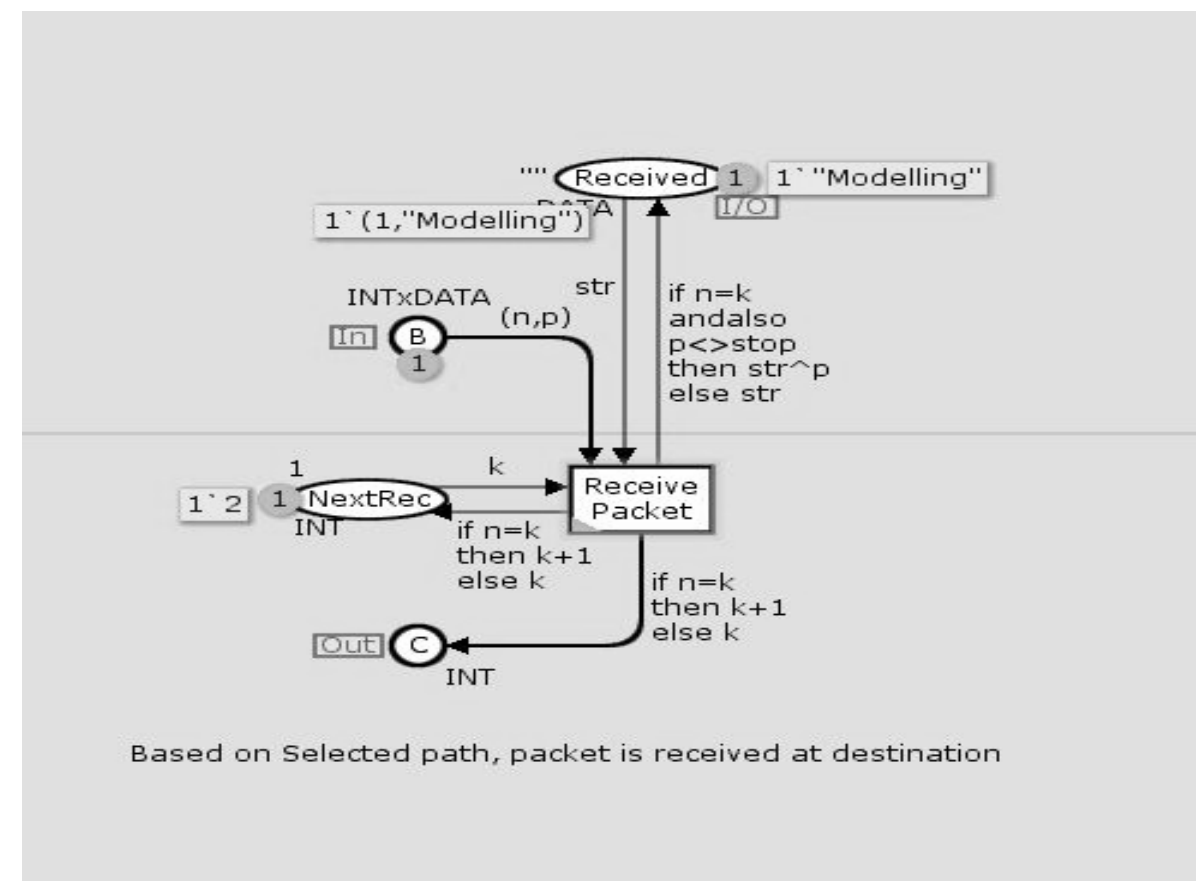

Figure 8. Energy Efficient Route Selection 


\subsection{OLSR verification:}

For verification of protocol, it is expected that when more than $70 \%$ packets are received at receiver end, then it is said protocol is realistic. From simulation report, total number of packets received and packets lost are obtained. Total numbers of simulation steps required are 205 for receiving all packets at 7 instances. The analysis is shown in Table 5 .

Table 5. Verification of modified protocol using Petri Net

\begin{tabular}{|l|c|c|}
\hline Number of instances & Packets received & Packets lost \\
\hline 1 & 7 & 3 \\
\hline 2 & 7 & 3 \\
\hline 3 & 6 & 4 \\
\hline 4 & 9 & 1 \\
\hline 5 & 8 & 2 \\
\hline 6 & 8 & 2 \\
\hline 7 & 8 & 2 \\
\hline Total (packets sent 70) & 53 & 17 \\
\hline
\end{tabular}

\section{CONCLUSION}

Our modified protocol, achieves better energy efficiency as compared to OLSR in terms of Number of node alive, indicating better network lifetime. For modified protocol, the improvement in Number of nodes alive for pause time and speed variation is due to multipath technique included in the protocol in addition to cost function and source routing. Modified protocol shows more energy efficient behavior for denser network. This is because in denser network there is better chance of balancing the traffic through many different energy efficient paths. Simulation time variation trials show that for better value of Number of nodes alive, it is desirable to have higher pause time and number of nodes, while no specific impact of speed.

In our Petri net representation, we have tried to verify modified protocol. In CPN model, as the transmitted packets by the sender are received correctly at the receiver, we confirm that, the modified protocol functioning is verified.

\section{REFERENCES}

[1] Thierry Plesse, Cedric Adjih, Pascale Minet, Anis Laouiti, Adokoe' Plakoo, Marc Badel, Paul Muhlethaler, Philippe Jacquet and Je'rome Lecomte, ( 2005) "OLSR performance measurement in a military mobile ad hoc network", Ad Hoc Networks 3, Elsevier, pp. 575-588.

[2] Taesoo Jun, Angela Dalton, Shreeshankar Bodas, Christine Julien, and Sriram Vishwanath, (2008) "Expressive Analytical Model for Routing Protocols in Mobile Ad Hoc Networks", IEEE international Conference on Communication, pp. 1-7.

[3] X.Hong, K.Xu and Gerla,(2002) "Scalable Routing Protocols for MANET”, IEEE network, Vol. 16, pp. 11-21.

[4] P. Sivasankar, C.Chellappan and S. Balaji, (2010) "Performance Evaluation of Energy Efficient On demand Routing Algorithms for MANET", 2008 IEEE Region 10 Colloquium and the Third ICIIS, Kharagpur, INDIA, pp. 1-5.

[5] Silvia Giordano \& Ivan Stojmenovic (2002) Mobile Ad Hoc Networks, Handbook of Wireless Networks and Mobile Computing, John Wiley \& Sons Publishers.

[6] Jiazi Yi, Eddy Cizeron, Salima Hamma and Benoît Parrein, (2008) "Simulation and Performance Analysis of MP-OLSR for Mobile Ad hoc Networks”, IEEE WCNC, pp. 2235-2240. 
International Journal of Wireless \& Mobile Networks (IJWMN) Vol. 3, No. 4, August 2011

F. Chiti, M. Ciabatti, G. Collodi, D. Di Palma, R. Fantacci, and A. Manes, (2006), "Design and application of enhanced communication protocols for wireless sensor networks operating in environmental monitoring”, Proceedings of IEEE International Conference on Communications (ICC '06), Istanbul, Turkey, Vol. 8, pp. 3390-3395.

[8] Geetha Jayakumar, and G. Gopinath, (2007) "Ad Hoc Mobile Wireless Networks Routing Protocols - A Review”, Journal of Computer Science, 3 (8), pp. 574-582.

[9] Ajit Singh, Harshit Tiwari, Alok Vajpayee and Shiva Prakash, (2010) “ A Survey of Energy Efficient Routing Protocols for Mobile Ad-hoc Networks", International Journal on Computer Science and Engineering (IJCSE), Vol. 02, No. 09, pp. 3111-3119.

[10] Saoucene Mahfoudh and Pascale Minet, (2008) “An energy efficient routing based on OLSR in wireless ad hoc and sensor networks", 22nd International Conference on Advanced Information Networking and Applications - Workshops IEEE Computer Society, pp. 1253-1259.

[11] C.-K. Toh, (2001) “ Maximum Battery Life Routing to Support Ubiquitous Mobile Computing in Wireless Ad Hoc Networks", IEEE Communication Magazine, pp. 138-147.

[12] Stephen Mueller, Rose P. Tsang and Dipack Ghosal, (2004) "Multipath Routing in Mobile Ad Hoc Networks: Issues and Challenges", Lecture Notes in Computer Science, Vol. 2965, pp. 209 -234 .

[13] Mustafa K. Gurcan, Hadhrami Ab Ghani, Jihai Zhou and Anusorn Chungtragarn, (2011) "Bit Energy Consumption Minimization for Multi-path Routing in Ad Hoc Networks”, Computer Journal, Volume 54, Issue 6, pp. 944-959.

[14] S. R. Biradar, Koushik Majumder, Subir Kumar Sarkar and Puttamadappa, (2010) "Performance Evaluation and Comparison of AODV and AOMDV", International Journal on Computer Science and Engineering (IJCSE), Vol. 02, No. 02, pp. 373-377.

[15] Thomas Clausen and Philippe Jacquet,(2010) “ The optimized Routing Protocol for Mobile Ad Hoc Networks: Protocol Specification”, INRIA, version 1, pp. 1-53.

[16] Sun Xuekang, Gu Wanyi, Xiao Xingquan, Xu Baocheng and Guo Zhigang, (2009) “ Node Discovery Algorithm Based Multipath OLSR Routing Protoco"1, WASE International Conference on Information Engineering, IEEE Computer Society, pp. 139-142.

[17] Floriano De Rango and Marco Fotino, (2009) "Energy Efficient OLSR Performance Evaluation under Energy aware Metrics”, SPECTS, pp. 193-198.

[18] T. Clausen and P. Jacquet, (2003), "Optimized Link State Routing Protocol (OLSR)”, RFC3626, pp. 1-75.

[19] Arati Manjeshwar and Dharma P. Agrawal, (2001) "TEEN: A Routing Protocol for Enhanced Efficiency in Wireless Sensor Networks", Fifteenth International Parallel and Distributed Processing Symposium (IPDPS’01) Workshops, Vol. 3, pp. 30189a.

[20] Alemneh Adane, (2008) Active Communication Energy Efficient Routing Protocol of Mobile Ad Hoc Networks (MANETS), PhD Thesis, Addis Ababa University, Computer Engineering, Ethiopia.

[21] IEEE Computer Society LAN MAN Standards Committee,( 1999) Wireless LAN Medium Access Control and Physical Layer Specifications, in IEEE 802.11 Standard.

[22] Radhika D. Joshi and Priti P. Rege, (2009) "Application Specific Node Selection Criteria for Mobile Ad Hoc Networks", CIIT International Journal of Wireless Communication, CIIT-IJ0386, Vol. 1, No. 6, pp. 274-283.

[23] Bor-rong Chen and C. Hwa Chang, (2003) “ Mobility Impact on Energy Conservation of Ad Hoc Routing Protocols”, Proceedings of International Conference on Advances in Infrastructure for Electronics, Business, Education, Science, Medicine and Mobile Technologies on the Internet (SSGRR'03) MTC, pp. 1-7. 
International Journal of Wireless \& Mobile Networks (IJWMN) Vol. 3, No. 4, August 2011

[24] Kathrin Hoffmann, (2010) "Formal Modeling and Analysis of Mobile AdHoc Networks and Communication Based Systems using Graph and Net Technologies", Bulletin of the European Association for Theoretical Computer Science (EATCS), No.101, pp.148-160.

[25] Amirineni, K K Chinara, S Rath, S K , (2010), "Validation of Clustering Algorithm for Mobile Ad-Hoc Networks Using Colored Petri Nets", Proceedings of the 4th National Conference; Bharati Vidyapeeth's Institute of Computer Applications and Management, New Delhi INDIACom, Computing For Nation Development.

[26] To-yat Chung, (1996) "Petri Nets for Protocol Engineering, Elsevier, Computer communications", pp. 1250-1257.

[27] Bruno Blaskovic, (1998) "Petri Net Modeling for Signaling Protocol Synthesis", Electro technical conference, Ninth Mediterranean, Melecon, pp. 706 - 710.

[28] Lisa Wells, (2006) "Performance Analysis using CPN Tools", ACM, Value Tools '06, Pisa, Italy, pp. 1-10.

[29] Lars M. Kristensen, Soren Christensen and Kurt Jensen, (1998) "The practitioner's guide to coloured Petri nets”, Springer Verlag, International Journal STTT, 2, pp. 98-132.

[30] Lars Michael Kristensen, Jens Bæk Jorgensen, and Kurt Jensen, (2004) "Application of Coloured Petri Nets in System Development", Springer-Verlag, ACPN 2003, LNCS 3098, pp. 626-685.

[31] Soren Christensen and Leif Obel Jepsen, (1991) "Modeling and Simulation of a Network Management System using Hierarchical Coloured Petri Nets", In Erik Mosekilde, Proceedings of the Simulation Multiconference, pp. 1-11.

[32] D.A. Zaitsev and T.R. Shmeleva, (2006) Simulating of Telecommunication Systems with CPN Tools, Students' book on the course - Mathematical Modeling of Information Systems, Transaction No. 5.

[33] Lars Michael Kristensen and Kurt Jensen, (2004) "Specification and Validation of an Edge Router Discovery Protocol for Mobile Ad Hoc Networks”, Springer-Verlag, INT 2004, LNCS 3147, pp. 248-269.

\section{Author 1}

Radhika D Joshi received the BE degree in 1993 from Pune University and ME Degree from Pune University in 2002. She is currently a PhD student at the University of Pune. She is pursuing research in the field of Wireless Mobile Ad hoc networks energy management issues. At present she is serving as Assistant Professor at Electronics and Telecommunication Engineering department of College of Engineering Pune, which is an autonomous institute of Government of Maharashtra. Her areas of interests are wireless communication, signal processing and electronic

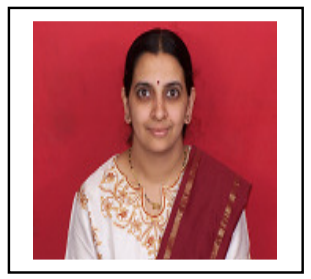
devices and circuits. She has received grants for two research proposals from two funding agencies recently. She has published several papers in journals and international conferences.

Author 2

Priti P. Rege received her Bachelor's and Master's Degrees from Devi Ahilya University Indore. She received a gold medal for her ME and received $\mathrm{PhD}$ from the University of Pune in 2002. She was recipient of "Nagarkar Fellowship" given by Dattatraya Pratisthan of Pune for carrying out research on: Subband coding of Images". In 1993, she joined Electronics and Telecommunication Engineering Department of College of engineering Pune, which is an autonomous institute of

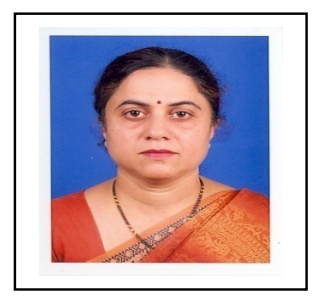
Government of Maharashtra. She is currently serving as Professor (Electronics Engineering) in the department of Electronics and Telecommunication Engineering. Her interests are in signal processing, image processing, simulation and modeling. Several of her papers have appeared in leading journals and conferences. 